\title{
REFLEXIONES SOBRE LA REPRESENTACIÓN ÓSEA DE INDIVIDUOS INFANTILES EN CONTEXTOS ARQUEOLÓGICOS: APORTACIONES Y LIMITACIONES METODOLÓGICAS
}

\author{
A Reflection on Infants Skeletal Remains in Archaeological Contexts: Limits and \\ Contributions in the Methodology
}

\author{
Isabel MOLERo Rodrigo \\ Investigadora independiente \\ isabel.iii@hotmail.com
}

Fecha de recepción: 17-II-2013

Fecha de aceptación: 8-III-2013

\begin{abstract}
Resumen: El estudio de los restos óseos infantiles procedentes de contextos arqueológicos ha estado tradicionalmente relegado a un segundo plano frente al análisis de individuos adultos. Afortunadamente, en las últimas décadas comienzan a integrarse en estos estudios, restableciéndose el papel cultural que jugaron en las sociedades del pasado. No obstante, esta falta de investigaciones ha dejado un vacío de información importante que debemos considerar a la hora de realizar futuras interpretaciones, sobre todo cuando realicemos comparaciones con grupos ya estudiados En este trabajo se pretende reflexionar sobre las contribuciones de este registro infantil y los problemas metodológicos que conlleva el estudio sociodemográfico y paleopatológico de unos restos de pequeño tamaño, de composición frágil, fácilmente alterables ante la actividad tafonómica, y que, por tanto, pueden pasar desapercibidos en el proceso de recuperación arqueológica.
\end{abstract}

Palabras clave: osteoarqueología, individuos infantiles, paleodemografía, problemas metodológicos, interpretación ósea.

AвSTRACt: Osteological studies related to infant bones from archaeological contexts have traditionally been shuffled aside in front of adult bones analysis. Fortunately, in the last decades they are being integrated, restoring their cultural role that played in past societies. However, this investigation bias had left an important lack of information, which should 
be regarded before considering future interpretations, moreover by the time of carrying out comparisons between groups already studied since the infant record might not been properly studied. This paper discuss the contributions from the study of these remains and several methodological problems implied in the study of little-size remains, with a weak bone composition, changeable because of taphonomical processes and, easily unnoticed during the archaeological recovery, also their difficulties in the estimation of sociodemographic and paleopathological parameters.

Keywords: ostheoarchaeology, infant bones, paleodemography, methodological problems, bone interpretation.

\section{INTRODUCCIÓN}

Los restos óseos humanos son una importante fuente para el conocimiento de las condiciones de vida de los integrantes de un grupo o población. Existen distintos parámetros que siempre aparecen o deberían aparecer en estudios de Antropología física, como la determinación del número mínimo de individuos, la edad, el sexo o las características antropológicas en base al estado de salud y de dieta y que, unidos al estudio arqueológico de los contextos materiales, la distribución espacial, los rasgos culturales o el entorno ecológico, entre otros, permiten dar una visión de conjunto sobre las características de una determinada comunidad o grupo. Es decir, a partir de la interpretación de todos estos resultados podemos acercarnos a la imagen de cómo eran los grupos humanos, a su estructura y sus modos de vida durante un periodo de tiempo concreto.

Dentro de los estudios osteoarqueológicos, los individuos infantiles han estado marginados durante años, quizás porque su identificación en el yacimiento puede resultar más complicada para arqueólogos no especializados en su anatomía. Además, los huesos sufren un mayor deterioro por su frágil composición química, siendo su recuperación más complicada dado que la mayor parte de los núcleos de osificación se encuentran sin fusionar. Por otra parte, el hecho de que la mayoría de los ajuares sea de menor entidad, hizo que muchos de los estudios tradicionales pasaran por alto su consideración. Afortunadamente, el panorama ha cambiado significativamente en las últimas décadas, si bien no debemos olvidarnos del vacío de información que éstos nos han dejado y que afectan al investigador, especialmente, a la hora de realizar estudios comparativos (De Miguel, 2010a).

La Antropología física se ha visto impulsada a raíz del aumento de trabajos de campo y, por tanto, por la proliferación del número de hallazgos. Dentro de esta situación, los restos humanos asociados a individuos infantiles han comenzado a ser mirados bajo lupa, formando en la actualidad, parte habitual de las investigaciones. Y es que, los valores de probabilidad de muerte en grupos jóvenes y ancianos son más elevados que en edades medias, independientemente de la estimación de esperanza de vida al 
nacimiento (Krenzer, 2006). De esta forma, la situación que cabe esperar, por ejemplo, de una necrópolis relativamente grande, es un elevado número de restos perinatales e infantiles jóvenes, así como de unos cuantos restos infantiles y algunos adolescentes. Ya simplemente teniendo en cuenta este hecho, es suficiente como para incluirlos dentro de las investigaciones bioarqueológicas (Baker, 2005). No debemos olvidar que entre un tercio y un cuarto de nuestra vida somos individuos subadultos (González, 2008).

No obstante, las dificultades en cuanto al estudio e interpretación no son pocas. Por tanto, en este artículo, se pretende reflexionar sobre la importancia de llevar a cabo estudios metódicos una vez conocidos los sesgos que el análisis del registro óseo puede conllevar. Comenzaré con un breve repaso historiográfico sobre la bibliografía tradicional, destacando algunas de las carencias principales tanto del método como del propio registro y, especialmente, sus contribuciones, es decir, sus límites y aportaciones principales en el estudio osteoarqueológico infantil. Así, nos vamos acercando hacia una construcción más objetiva de la concepción histórica de la infancia. A través del análisis exhaustivo de estos restos óseos, podemos llegar a conocer aspectos como las tareas que desarrollaron, si existían diferencias significativas por sexos, o incluso la edad en la que culturalmente dejaban de ser nińos. En definitiva, acercarnos al conocimiento de cómo la sociedad del pasado entendió la infancia.

\section{REFLEXIÓN TERMINOLÓGICA}

Lo primero que debemos preguntarnos es qué características definen a la infancia. En la actualidad, entendemos por ella el periodo de la vida humana desde el nacimiento hasta la pubertad. Se puede considerar, desde el punto de vista biológico, que una vez que se ha cerrado del todo la sutura esfenobasilar (unión del hueso esfenoides con el occipital, a partir de los 15 años aproximadamente) o ha erupcionado el tercer molar, también conocida como "muela del juicio» (si bien ésta puede no emerger por ser un resto atávico de nuestra evolución), el individuo ha entrado en la etapa adulta. Pero en lo que se refiere a la interpretación cultural sobre la infancia, ésta dista mucho que ser la compartida en otros momentos históricos. Se trata, por tanto, de un concepto impreciso (Chapa, 2008).

Después de nacer, nuestra historia biológica puede estar dividida en cinco etapas, a saber: infancia, niñez, adolescencia, juventud y edad adulta, siendo los cambios en la velocidad de crecimiento los que mejor definirán estas transiciones a través de procesos biológicos (Bogin, 1997). El problema es que, aunque éstos quedan reflejados en el registro fósil, no ocurre lo mismo con la interpretación cultural. Sin entrar en esta problemática, la nomenclatura empleada en este trabajo será la relacionada con los procesos biológicos de crecimiento. 
Durante la década de los años sesenta comenzaron a aparecer los primeros trabajos en Antropología física centrados en el estudio de estos restos, en contextos arqueológicos, teniendo en cuenta los posibles condicionantes sociales y ambientales, a partir del análisis de sociedades contemporáneas. De esta forma se dieron cuenta de que si conseguían demostrar la validez en la comparación de datos de los grupos actuales con los históricos, se podría desarrollar una aproximación paralela en las muestras arqueológicas. En 1962, W.M. Krogman elabora un libro dedicado a la osteología forense donde destaca un capítulo exclusivo en el que recoge los métodos que hasta el momento existían para la estimación de edad en individuos infantiles; y en 1978, Fazekas y Kósa, preocupados también por los ritmos de crecimiento esqueléticos, construyen una serie de funciones para su estimación en la edad fetal (Rissech, 2008).

Pero, asociado a este esperanzador panorama y a la proliferación de nuevos hallazgos, la lista de dificultades no parecía desaparecer, haciendo que el valor de las conclusiones alcanzadas no fuera la deseada. Esta situación se mantendría en los siguientes trabajos publicados, en los que podemos observar aún un análisis generalista de estos restos, que desde un punto de vista teórico eran nombrados bajo el término «subadulto», pareciendo aventurar un cierto desdén en el estudio de los restos humanos en comparación con el de los adultos. Esta misma falta de atención se manifestaba en la preocupación de la Antropología física tradicional en el estudio del esqueleto craneal a expensas del postcraneal, como vemos según los títulos de los trabajos publicados.

Considerados tradicionalmente como miembros pasivos de la sociedad, ajenos al desempeño de posibles roles sociales, las referencias históricas indican que estos individuos infantiles accederían pronto a las funciones sociales con plenos derechos. Esta importancia se desprende de las prácticas de cuidado registradas en múltiples yacimientos, destacando las sepulturas de la Edad del Bronce en el Cerro de la Encina (Monachil, Granada), donde la integración de juguetes como ajuar funerario, relegada durante mucho tiempo al terreno de la simple curiosidad, puede ser entendida como parte del proceso de aprendizaje y de socialización (Sánchez, 2008).

Pero, antes de proceder a la valoración de este registro óseo, debemos de tener en cuenta las siguientes limitaciones si queremos ser lo más objetivos posible en cuanto a la aportación de la Antropología física en el estudio de los individuos infantiles.

\section{INFORMACIÓN ANTROPOLÓGICA I: PROBLEMAS EN LA DETERMINACIÓN DEL SEXO}

Cuando tratamos de determinar el sexo en individuos infantiles nos encontramos con un problema directamente relacionado con la edad, puesto que la sinostosis (unión ósea) de determinados elementos no ha tenido aún lugar, ya que las características que 
definen el sexo no aparecen hasta la pubertad. Además, existen factores socioecológicos como la alimentación, el clima o las enfermedades, que pueden influir en su desarrollo, modificando el aspecto que deberían presentar los huesos. En este sentido, sólo los estudios de ADN nucleico nos ofrecen una información más fiable, aunque no parece que sea un método fácilmente accesible en la actualidad para la mayoría de especialistas debido a su alto coste económico.

Y aunque existe una gran variedad de métodos de adscripción sexual para estos individuos, desafortunadamente no existen garantías aceptables para cualquier grupo cultural (Bruzek y Schmitt, 2008; González, 2008). Aun así, podemos considerar la validez de determinados estudios antropológicos de carácter mofognóstico (carácter cualitativo) sobre muestras representativas, bien conservadas, en los que se ha podido identificar con cierto grado de rigor la pertenencia a un determinado sexo (Aranda, 2008; Jiménez-Brobeil, 2008), centrándose en la cintura pelviana como mejor hueso diagnosticador del mismo (Fazekas y Kósa, 1978).

Para Schutkowski (1993), por ejemplo, las bases diferenciadoras del ilion en niños se reflejarían en la presencia de un ángulo en la escotadura ciática más angosto que en las niñas, cercano a los $90^{\circ}$, con una curvatura de la cresta ilíaca más pronunciada y, atendiendo a la morfología de la mandíbula, un mentón prominente, cuadrangular y más ancho. Pero estos índices no parecen mostrar diferencias significativas entre los dos sexos, solapándose los resultados y por tanto, pudiendo reflejar un sesgo importante en el análisis de nuestros resultados. No podemos olvidarnos de que el margen de error sigue siendo muy amplio, más aún si la preservación del registro no es óptima.

Por otra parte, en los últimos años se está teniendo en cuenta el cálculo de funciones discriminantes a partir de las medidas de uno o varios huesos, normalmente huesos largos, válido, sobre todo, para aquel material que se encuentre fragmentado o para aquellos registros en los que no se disponga de pelvis o mandíbula, es decir, para casos dudosos. Estos parámetros se obtendrían de la medida de huesos de individuos bien diagnosticados del mismo grupo poblacional o, en su defecto, de poblaciones similares. Además, se comienzan a valorar otros rasgos, como las dimensiones de la dentición temporal (Rodríguez Flórez, 2008) o de los metacarpos (Barrio, 2006), entre otros.

$Y$ es que, la relevancia de obtener el sexo de los individuos infantiles conservados nos permitiría acceder a datos de tipo sociocultural altamente relevantes a la hora de interpretar su presencia en los distintos contextos arqueológicos, aportando información sobre el tratamiento funerario y las distintas tipologías de enterramientos. Es decir, nos acerca al conocimiento de un tema tan interesante como es el de la mortalidad diferencial en sus primeras etapas de vida, lo que podría abogar en ideas que confirmasen la existencia de unas prácticas culturales que favorecieran a uno u otro sexo. Es ésta una 
meta que la osteoarqueología se viene marcando desde sus orígenes puesto que a la hora de hacer inferencias de tipo cultural no viene a significar lo mismo la presencia de individuos de uno u otro sexo.

\section{INFORMACIÓN ANTROPOLÓGICA II: PROBLEMAS EN LA ESTIMACIÓN DE LA EDAD}

Otro de los problemas y desafíos metodológicos es el de la estimación de la edad de muerte. No obstante, al contrario de lo que ocurre con su estimación para individuos adultos, los criterios son más precisos para etapas infantiles debido a la remodelación ósea constante desde el nacimiento (Saunders, 1992). Y es que, la sola observación de la dentición, así como de gran parte de los restos esqueléticos, va a permitir que en un primer análisis distingamos fácilmente entre individuos adultos y sujetos infantiles. Más complicado resultará precisar su edad.

Los estudios se basan, sobre todo, en las mediciones y metamorfosis del esqueleto craneal y/o postcraneal ya que las líneas de fusión de los huesos siguen siendo visibles hasta dos años después de la sinostosis. Dado que la unión parece iniciarse antes en individuos femeninos, existiendo ligeras diferencias entre el inicio y final de la sinostosis en ambos sexos, resulta importante conocer de antemano, siempre que sea posible, la adscripción sexual del individuo a estudiar. A pesar de ello hemos de tener siempre en cuenta la variabilidad, no sólo entre grupos sino también entre individuos, ya sean del mismo sexo, sobre todo si estamos trabajando con distintos periodos cronológicos.

Además, no podemos olvidarnos que, al igual que ocurría con el sexo, a edades tan tempranas su estado de salud, dieta, clima, patologías, etc., puede llegar a influir en la morfología del esqueleto alterando la información relativa a la edad y, por tanto, la precisión de la misma (De Miguel, 2010b). Todo ello hará que tengamos que aceptar rangos de variación amplios para cada etapa, siendo razonable de dos años en los primeros cinco de edad y para la pubertad, así como de cuatro ańos para los diez siguientes (Sundick, 1977).

Siguiendo la división de etapas de desarrollo tanto del esqueleto craneal y/o postcraneal como de los dientes, podemos observar una primera etapa de edad en la que aparecen la mayoría de los primeros centros de osificación y que se desarrollará entre el nacimiento y los cinco ańos aproximadamente, momento en que erupcionan los dientes deciduales y se calcifican los permanentes (Krogman e Iscan, 1986). Seguida de una segunda etapa en la que se incrementa el tamaño de los huesos, cambiando su configuración y forma, y finalizando con la unión de los huesos del acetábulo y la erupción de los dientes permanentes (a excepción del tercer molar) y que va desde los cinco hasta los trece años, con un rango de seis meses. Y un tercer momento de unión epifisal de los 
huesos largos, de la sínfisis púbica y de las costillas, entre los trece y los veinte años, también con un rango de aproximadamente seis meses (Krenzer, 2006). Para su estimación son de referencia obligada las tablas de desarrollo de los distintos huesos de Scheuer y Black (2000).

Pero, dentro de los diferentes métodos de estimación, el parámetro más uniforme y con mayor grado de precisión será el grado de erupción dental. Los dientes, por su morfología y estructura, son extremadamente resistentes al paso del tiempo. El método de calcificación de Ubelaker (2007), inspirado en el de Schour y Massler (1941) es el recomendado por el Workshop of European Anthropologist (WEA), y es que siempre que un diente no esté desarrollado del todo, dará una estimación de edad con cierta precisión (Ferembach, 1979).

En cuanto al uso que se ha dado al método morfométrico (estimación de la edad a partir de la medida, principalmente, de distintos huesos largos), no debería ser empleado nunca como único criterio, sino como método adicional que contraste la información obtenida a través de la combinación de los métodos descritos anteriormente. Por un lado, debido a las diferencias entre sexos y poblaciones, y por otro, por las distintas situaciones medioambientales y de la nutrición que afectan al desarrollo óseo, sobre todo, a las longitudes máximas de los huesos largos.

Pero el gran problema que limita estos análisis es la falta de un consenso metodológico. El que encontremos distintas edades utilizando uno u otro método refleja que las poblaciones que se usan como material de referencia presentan distintos ritmos de calcificación. Tras varios años de utilización se han registrado graves dificultades ya que la inclusión del rango de variación superpone unos grupos de edad con otros. Por otra parte, la precisión de la edad, al ser más complicada para aquellos individuos infantiles en los que se desconoce el sexo, va a requerir en muchas ocasiones que crucemos distintas metodologías, es decir, que sigamos un método mixto en el que se tengan en cuenta el grado de sinostosis de los principales centros de osificación, la edad morfométrica y la erupción dental de un mismo individuo, siempre y cuando la preservación del registro óseo lo permita. Por último, no podemos olvidarnos de que la edad a estimar será la edad biológica o esqueletal, y por tanto, no necesariamente igual a la edad cronológica.

\section{PROBLEMAS DE REPRESENTACIÓN Y AFECTACIÓN TAFONÓMICA}

A la hora de explicar un elevado número de individuos inmaduros en necrópolis, hemos de tener en cuenta dos hechos cotidianos: los nacimientos tempranos y los desarrollos lentos. El hecho de que en la actualidad, la proporción de inmadurez en contextos funerarios sea mayor, no quiere decir que ésta sea real sino que, como ya hemos visto, desde hace unas décadas, comenzaron a tenerse en cuenta en los estudios funerarios 
los datos antropológicos y especialmente, de individuos infantiles. Hasta entonces, su fragilidad y por tanto, facilidad de fragmentación, así como la dificultad de su estudio, hizo que quedaran al margen de la atención de algunos investigadores. Siempre que las condiciones lo permitan, se puede documentar hasta un $60 \%$ de los restos de inmaduros (Ferembach, 1962) o incluso un 80\% (Alesán y Magosa, 1996) sobre el total. Lo que se desprende de ello es que el número de individuos infantiles en poblaciones en contexto arqueológico es alto y, si se diera el caso contrario, deberíamos atender a los siguientes sesgos tafonómicos.

El momento de recogida de los restos óseos se debe realizar de manera exhaustiva ya que, al no haber finalizado su desarrollo presentan las epífisis sin fusionar, las cuales, por su reducido tamaño, son fácilmente confundibles con el sedimento arqueológico pudiendo presentar además su estructura modificada por una mala conservación, ya sea por la presencia de concreciones, hongos, bacterias, levaduras en los conductos de Havers, etc., o incluso por fracturas debido a la presión sedimentaria y/o presencia de raíces, dificultando su estudio y sus resultados.

Entre estos problemas, es de destacar la humedad del yacimiento, la cual perjudica gravemente su preservación, partiendo de que el hueso de un adulto es más estable ya que se encuentra más cristalizado. Nos vamos a encontrar, con un problema de preservación diferencial, es decir, los huesos de individuos infantiles al tener poco volumen y mayor superficie, dado que la parte en la que se realiza el contacto con el medio es esta superficie, van a encontrarse más influenciados por los condicionantes ambientales. Y lo mismo sucede con el tiempo transcurrido después del nacimiento, no siendo lo mismo que el niño haya nacido muerto o haya muerto poco después de nacer ya que en esas pocas horas de vida, si éste ha sido alimentado, comenzará a formar su flora intestinal. En este caso, la descomposición iniciada por las bacterias será de dentro hacia fuera (González, 1999).

Si añadimos el problema de la acidez del suelo, la probabilidad de conservación será aún menor, ya que cualquier medio ácido, por duro que sea el tejido esquelético, tenderá hacia su desaparición, ya sean individuos infantiles o adultos. Aun así se ha conseguido recuperar en medios ácidos alguna corona de diente (dado que el esmalte dental es el tejido más duro del esqueleto humano) pero para ello se ha de tener en cuenta una metodología de excavación especialmente rigurosa para evitar confundirlos con el mismo sedimento arqueológico. En estos casos, es de especial ayuda el registro fotográfico in situ y la posterior extracción arqueológica en bloque, ya que la descamación y fragmentación ósea a la hora de su recogida, puede llegar a imposibilitar el diagnóstico antropológico o incluso patológico de los mismos.

Todo ello debe ser tenido en cuenta, particularmente, a la hora de interpretar aquellas necrópolis donde se hayan documentado fosas expresamente realizadas para 
individuos infantiles y donde no se encuentren restos en su interior, es decir, que se manifiesten aparentemente vacías, pudiéndose interpretar erróneamente como inhumaciones de tipo exclusivamente simbólico. En estos casos, se requiere de un exhaustivo tamizado del sedimento por si aparecieran pequeños fragmentos de huesos que pudieran confundirse con el mismo, especialmente cuando se trata de huesos sesamoideos (que aparecen concretamente en las articulaciones de manos y pies, sirviendo de protección de los tendones).

En definitiva, nos encontramos con una amplia cantidad de variables que se pueden ver implicadas en los contextos arqueológicos y que debemos tener presente antes y durante el trabajo de recuperación y estudio del material.

\section{INFORMACIÓN PALEOPATOLÓGICA}

La Paleopatología se encarga del estudio de las huellas registradas en los restos óseos humanos producidas por las enfermedades desarrolladas en el pasado. Estas marcas permiten evidenciar los padecimientos que sufrieron los individuos durante su vida, así como su dieta o incluso la posible causa de su muerte, si bien la mayoría de las veces no podemos discernir las enfermedades que padecieron estos individuos y/o las causas que provocaron su muerte. A veces una infección es lo suficientemente grave cuando no se tienen recursos para combatirla. Muy probablemente fue ésta la causa de muerte de los nińos que encontramos en los yacimientos. Más frecuentemente, se encuentran signos que pueden asociarse con una u otra enfermedad, sobre todo en lo que se refiere a enfermedades infecciosas o carenciales, aunque difícilmente pueden ser relacionadas directamente con la muerte de éstos.

Poder identificar los signos patológicos en el registro va a estar condicionado por el proceso de desarrollo de la misma enfermedad ya que éstas, al margen de los procesos traumáticos, sólo dejan señales óseas cuando su desarrollo es largo. Además, el modo en que un organismo responde ante una lesión ósea es limitado (Salter, 1976), y las alteraciones no siempre tendrán su origen en una determinada enfermedad sino que pueden encontrarse relacionadas con varias a la vez, limitando los diagnósticos. Entre las patologías más frecuentes en individuos infantiles podemos encontrar aquellas de tipo carencial, con una implicación posiblemente metabólica y que quedan reflejadas a nivel óseo tras un periodo de tiempo continuado.

Puesto que en las primeras etapas de vida el individuo se encuentra expuesto a una gran variedad de agresiones ya sean de origen infeccioso como medioambiental, su sistema inmunitario tendrá que reaccionar. En una lactancia deficitaria o un destete precoz, puede darse un sistema inmunitario débil, en el que los niños quedan ampliamente expuestos a distintos riesgos. Desde el punto de vista de la epidemiología, las infecciones 
provocan mayor número de muertes, ya sea en individuos infantiles como en adultos. El problema, como ya se ha dicho, es que son escasas las veces en que éstas dejan evidencias sobre los restos óseos porque para ello sería necesaria una evolución crónica y lenta de la infección, hecho menos frecuente que las infecciones más comunes que comprometen la vida más rápidamente. Por tanto, la probabilidad de que dejen improntas en el registro óseo es muy reducida, aunque se han documentado en alguna ocasión en necrópolis donde encontramos inhumaciones simultáneas de individuos infantiles. Siempre que se descarte una muerte por acción traumática, podemos pensar que la muerte de estos individuos pudo ser provocada por algún tipo de infección. Por el contrario, si el proceso de la infección fuera largo, sí encontraríamos huesos afectados por patología. Se han dado casos, como en la necrópolis mudéjar de Crevillent (De Miguel, 2010b), en los que los senos maxilares están afectados por signos de sinusitis o, incluso lesiones costales con periostitis en la cara interna tal vez en relación con un proceso de tuberculosis pulmonar tras el contagio producido por el Bacilo de Koch, o signos de osteomielitis, enfermedad conocida desde el Neolítico (Brothwell, 1981) y que afecta a la zona esponjosa del hueso causando hinchazones y lesiones donde penetran los microorganismos (Krenzer, 2006). Añadido a todo ello, las complicaciones durante el embarazo podrían justificar de por sí el elevado índice de mortalidad, así como ocurre en la actualidad en países con escaso desarrollo sanitario y de higiene, a lo que podemos sumar la proliferación de infanticidios en determinados momentos históricos en función de su contexto cultural, sus circunstancias familiares y/o motivos religiosos.

Dentro de las patologías de origen metabólico, destacarían enfermedades como la criba orbitaria (por problemas anémicos), raquitismo (que produce un retraso general del crecimiento óseo y deformidad), cuya evidencia es escasa a pesar de la elevada frecuencia con la que se ha manifestado hasta época reciente (tal vez debido a la escasez de estudios sobre individuos infantiles) o la hipoplasia del esmalte dental. Esta última consiste en una deficiencia del espesor del esmalte debido a la interrupción de la mineralización del mismo (amelogénesis) determinada por factores como la malnutrición o posibles infecciones apareciendo en forma de líneas horizontales irregulares en la superficie del esmalte, fácilmente visibles en la cara labial de la corona. Suelen aparecer con mayor frecuencia en el momento crítico del destete, cuando el individuo pasa a ingerir alimentación sólida con alto contenido en hidratos de carbono. Estos estudios resultan muy interesantes si queremos tratar el tema de la alimentación en distintas sociedades a lo largo del tiempo, así como la incidencia de la presión ambiental ya que, conociendo la edad aproximada de calcificación de la pieza dentaria en cuestión, se puede analizar en qué momento este individuo sufrió la afección con gran precisión, ya que estas líneas van a permanecer inalterables en el diente. Otra de las patologías documentadas sería la presencia de caries y/o sarro, no tan frecuente en individuos infantiles como en etapas más avanzadas si bien no es extraño encontrar este tipo 
de patología oral asociado a dietas ricas en hidratos de carbono y con una deficiente higiene bucal (Campillo, 2001).

$\mathrm{Y}$ en cuanto a las malformaciones óseas infantiles, aunque son poco frecuentes (dentro del contexto arqueológico espańol), se han podido identificar casos tales como la fusión costal en la maqbara de la Plaza del Castillo (Pamplona, Navarra) o en el cementerio visigodo del Tolmo de Minateda (Hellín, Albacete) aunque en ninguno de estos dos casos la mera fusión ósea justificaría de por sí la muerte de los individuos (De Miguel, 2010a). En cuanto a las trepanaciones destaca el conocido caso del yacimiento de La Sarsa (Bocairent, Valencia), adscrito al Neolítico Cardial y que presenta un fragmento de parietal con parte de una trepanación realizada siguiendo el método de barrenado (Campillo, 2007; García, 1983). Dado que este fragmento no presenta regeneración ósea se ha interpretado como un individuo que no pudo sobrevivir a la trepanación, si bien no se pudo precisar si la misma fue realizada ante o post-mortem y menos aún, el motivo de su realización.

Más frecuentes en los niños son las denominadas fracturas «en tallo verde» en la que se produce una fractura parcial debido a la elasticidad de la cortical del hueso largo y una reparación del mismo, sin alterar su forma, dada la capacidad de regeneración ósea tan elevada para esta etapa (Salter, 1976; Charlier, 2008b). También se encuentran cráneos con erosiones, probablemente asociadas a traumatismos de distinta gravedad (lo más normal es que sean casos leves y que, por tanto, no conlleven grandes problemas de salud).

Por otra parte, es interesante destacar las marcas registradas en lugares de inserción muscular, conocidas como entesopatías, ya que éstas nos permiten considerar tareas físicas reiteradas en función del músculo afectado y, por tanto, establecer a partir de aquí distintas hipótesis de tipo cultural sobre el rol de éstos en la sociedad. El hueso es un tejido mineralizado, duro y resistente pero, a pesar de esta dureza, es maleable por lo que, ante la influencia de algunos factores de estrés físico, como las cargas de peso repetidas sobre una determinada parte del cuerpo (cuya incidencia variará en función de la edad y la arquitectura del hueso) responde a estos estímulos modificando su morfología, en concreto en la zona de inserción del tendón en el hueso (Malgosa, 2003). Dentro de estas modificaciones, podríamos destacar la elevación del borde ancóneo del cúbito relacionada con la extensión de la articulación húmero-cúbito y que está asociada a soportar objetos pesados meciéndolos con los dos brazos; o entesopatías en las tuberosidades calcáneas por la repetición de acuclillamientos. Es decir, a partir de estos análisis de marcadores físicos podemos desarrollar inferencias acerca de posibles trabajos y su relación con determinados rangos de edad en determinados momentos cronoculturales. 


\section{CONCLUSIONES}

Como hemos visto, el número de restos esqueléticos infantiles en una necrópolis que no presente agresiones significativas, es decir, donde el grado de preservación sea óptimo y esté dentro de un perfil de sociedad en crecimiento, tenderá a ser elevado, ya que la mortalidad en esta etapa es alta como resultado de la incidencia de enfermedades infecciosas y las complicaciones durante y tras el parto. Por lo que, si ocurriera la situación contraria, deberíamos considerar factores como los ya vistos, el reducido tamaño de los huesos que los hace fácilmente confundibles con el sedimento, o que los mismos hayan sido recuperados parcialmente por una persona que desconozca la anatomía de los individuos infantiles, la humedad del medio, la acidez del suelo o incluso el tiempo transcurrido tras la muerte del individuo. Dentro de los principales problemas que ya destacamos, podemos encontrar, especialmente en lo relativo a la recogida del material, o su posterior estudio comparativo, importantes sesgos que pueden oscurecer los resultados. Para evitar que su incidencia sea mayor, la recuperación del registro óseo ha de ser exhaustiva, especialmente tratándose de huesos pertenecientes a individuos infantiles que aún no han finalizado su desarrollo, resultando fundamental el registro fotográfico y el conocimiento de la anatomía de los esqueletos inmaduros por parte de quienes realizan la labor de recuperación del material (González, 2008).

Una vez que los restos han sido trasladados al laboratorio para su estudio, tendremos que considerar una serie de apartados que siempre deben aparecer, como la adscripción sexual o la edad, es decir, los parámetros paleodemográficos básicos, si bien las dificultades de su estimación no son pocas. El margen de error a la hora de determinar el sexo de individuos infantiles es muy amplio, aunque las inferencias que podemos extraer de estas conclusiones pueden resultar muy interesantes, sobre todo a la hora del estudio diferencial de las inhumaciones. Pero, ante todo, si la conservación y representación de los restos no es la adecuada y, si no disponemos de un apoyo económico suficiente para realizar análisis de $\mathrm{ADN}$, será conveniente dejar en incógnita su adscripción para evitar falsear la información. La edad biológica, al contrario, tiene un rango de error menor, siendo más precisa cuanto más joven es el individuo, ya que en estos momentos las modificaciones son más rápidas y constantes. Pero también los condicionantes ecológicos y sociales, así como las paleopatologías o la misma diversidad entre individuos, pueden afectar a esta estimación, por lo que es imprescindible reflejar la edad con unos aproximados márgenes de error adecuados.

Por su parte, el análisis de las patologías, siempre que la duración de las enfermedades ha sido lo suficientemente prolongada como para dejar marcas en los huesos, nos permitirá realizar consideraciones de estrés físico, siendo las entesopatías uno de los marcadores que mejor reflejan el grado de implicación de los individuos en la sociedad, 
y nos permiten conocer el tipo de trabajo desempeñado por estos individuos infantiles, concluyendo en el modo de integración social de este colectivo en el grupo.

Estas dificultades, ya vengan de la preservación ósea o de la metodología a aplicar no deben desalentarnos sino por el contrario, ser un factor que nos incite a estudiarlos con más detenimiento y, a la búsqueda de nuevas soluciones. Este artículo por tanto, pretende no sólo destacar el estado aún deficitario en el que se encuentra el análisis óseo infantil sino ser un punto de reflexión si vamos a realizar comparaciones con estudios elaborados décadas atrás y que no hayan sido revisados, así como a la hora de desarrollar futuros trabajos siendo lo más coherentes posibles en función del sesgo que presenten.

Gracias al estudio osteoarqueológico podemos llegar a reconstruir y comprender una buena parte de nuestro pasado biológico e histórico aunque, los debates en torno a su análisis e interpretación sean múltiples y de diversa índole, especialmente debido a la inexistencia de modelos de investigación específicos para este grupo en cuestión (Sánchez 2007), lo que hace que la precisión de los resultados no sea la deseada. No obstante, podemos conocer su representación sobre el total del grupo o población a través de su localización en las necrópolis, si recibieron un tratamiento especial tras su muerte y si por ende, este tratamiento puede inferirse al resto de miembros o no. Las ventajas de su estudio son, por tanto, dignas de consideración. Encontrar y estudiar individuos infantiles en yacimientos arqueológicos es de por sí relevante, por tanto, si se quiere conocer cuál era su grado de adaptación, y si disfrutaban del derecho de participar en los rituales funerarios (Bocquet-Appel, 2005).

No obstante, aún no resulta fácil llegar a conclusiones inequívocas en torno al papel que desempeñó la infancia en una sociedad en concreto. Quedan muchos aspectos por ser aclarados dentro del conocimiento de nuestro pasado biológico y su inclusión en la realidad cultural, por lo que se hace imprescindible el estudio de nuevos hallazgos, retomar antiguas y nuevas discusiones, así como la plena integración de la Antropología física en los estudios arqueológicos y culturales.

\section{BIBLIOGRAFÍA}

ALESÁN, A. y MALGOSA, A. (1996): «Las series subadultas de las necrópolis de Son real y S'illot des Porros (periodo talayótico, Mallorca). Nuevos datos», Revista Española de Antropología Biológica, 17, pp. 37-47.

ARANDA, G., et al. (2008): «El poblado y necrópolis argáricos del Cerro de la Encina (Monachil, Granada): las campañas de excavación de 2003-05». Cuadernos de Prehistoria de la Universidad de Granada, 18, pp. 219-264. 
BARRIO, P. A., et al. (2006): «Metacarpal sex determination in a spanish population", Journal of Forensic Science, 51 (5).

BAKER, B. J, et al. (2005): The osteology of infants and children. Texas A\&M university anthropology series 12 .

BOCQUET-APELL, J.P. (2005): La paléodémographie. Objets et méthodes en Paléoanthropologie. Comité des Travaux Historiques et Scientifiques, Paris, pp. 271-314.

BOGIN B. (1988): Patterns of human growth. Cambridge, Cambridge University Press.

BUZEK, J. y SCHMITT, A. (2008): «L'identification du sexe d'un individu à partir du squelette. Ostéo-archéologie et techniques médico-légales tendances et perspectives.» Manuel pratique de paléopathologie humaine, Paris, pp. 259-267.

CAMPILLO, D. (2001): Introducción a la paleopatología. Bellaterra, Barcelona.

CAMPILLO, D. (2007): La trepanación prehistórica. Bellaterra, Barcelona.

CHAPA, T. (2008): «Presencia infantil y ritual funerario». Nasciturus, Infans, Puerulus vovis mater terra. SIAP-Diputació de Castelló, Castelló, pp. 619-641.

CHARLIER, P. (2008a): «Lidentification du travail infantile en paléopathologie. Ostéo-archéologie et techniques médico-légales tendances et perspectives». Manuel pratique de paléopathologie humaine, Paris, pp. 309-310.

CHARLIER, P. (2008b): «Des lésions traumatiques sur un squelette d'immature: enfant battu, enfant malade ou enfant turbulent? Ostéo-archéologie et techniques médico-légales tendances et perspectives». Manuel pratique de paléopathologie humaine, Paris, pp. 311-313.

DE MIGUEL IBÁNEEZ, Ma.P., (2010a): «La infancia a través del estudio de los restos humanos desde el Neolítico a la Edad del Bronce en tierras valencianas». Restos de vida, restos de muerte, Museo de Prehistoria-Diputación de Valencia, Valencia, pp. 155-166.

DE MIGUEL IBÁÑEZ, Ma.P. (2010b): «Una visión de la infancia desde la osteoarqueología: de la Prehistoria reciente a la Edad Media». Complutum, vol. 21 (2), pp. 135-154.

FAZEKAS, I. y G.; KOSÁ, F. (1978): Forensic fetal osteology, Akademiai Kiadó Budapest. 
FEREMBACH, D., et al. (1979): «Recommandations pour déterminer l'âge et le sexe sur le squelette». Bull. Mém. Soc. Anthrop. Paris 6, pp. 7-45.

FEREMBACH, D. (1962), La nécropole Epipaléolithique de Taforalt, Casablanca, Rabat.

GONZÁLEZ MARTÍN, A. (2008): «Mitos y realidades en torno a la excavación, el tratamiento y el estudio de los restos arqueológicos no-adultos.» Nasciturus, Infans, Puerulus vovis mater terra, SIAP Diputació de Castelló, Castelló, pp. 57-76.

GONZÁLEZ MARTÍN, A., et al. (2012): «La evolución de «los niños»: algunas reflexiones sobre el registro fósil infantil en Paleoantropología,» Cadernos do GEEvH 1 (1), pp. 17-31.

GONZÁLEZ MARTÍN, A. (1999): Infancia y adolescencia en la Murcia musulmana. Estudio de restos óseos. Tesis doctoral, Universidad Autónoma de Madrid, Facultad de Ciencias, Departamento de Biología, Unidad de Antropología.

JIMÉNEZ-BROBEIL, S.A., et al. (2008): «Salud y enfermedad en Motilla de Azuer: una población de la Edad del Bronce de La Mancha». Revista Española de Antropología Física, 28, pp. 57-70.

JOHNSTON, F. E. (1968): Growth of the skeleton in earlier peoples, the skeletal biology of earlier human populations. London, pp. 57-66.

KRENZER, U. (2006): Compendio de métodos antropológico forenses para la reconstrucción del perfil osteo-biológico, Guatemala: CAFCA.

KROGMAN, W.M. e ISCAN, M.Y., (1986): The Human Skeleton in Forensic Medicine. Charles C. Thomas Publishers, Springfield.

MALGOSA, A. (2003): «Marcadores de estrés ocupacional». En Isidro A, Malgosa A., Paleopatología. La enfermedad no escrita. Ed. Masson S.A. Barcelona, pp. 221-235.

RISSECH, C. (2008): «Estimación de la edad biológica de los restos subadultos». En: Gusi-Gener F, Muriel S, Olária C (eds.) Nasciturus, infans, puerulus vobis mater terra: la muerte en la infancia. Servei d'Investigacions Arqueològiques i Prehistòriques, Diputació de Castelló, Castelló, pp. 72-92.

SALTER, R.B. (1976), Trastornos y lesiones del sistema musculoesquelético. Salvat, Barcelona. 
SÁNCHEZ ROMERO, M. (2007): «Actividades de mantenimiento en la Edad del Bronce del sur peninsular: El cuidado y la socialización de individuos infantiles». Complutum, 18, pp. 185-194

SÁNCHEZ ROMERO, M. (2008): «Childhood and the Construction of Gender Identities through Material Culture». Childhood in the Past 1, pp. 17-37

SAUNDERS, S. R. (1992): «Subadult skeletons and growth related studies», Skeletal biology of past peoples. Research methods, New York, pp. 1-20

SCHUTKOWSKY, H. (1993): "Sex determination of Infant and Juvenile Skeleton I. Morphognostic Features». American Journal of Physical Anthropology, 90, pp. 199-205.

SUNDICK, R. I. (1978): «Human skeletal growth and age determination», Homo 29, pp. 228-249

SCHEUER, L. y BLACK, S. (2000): Developmental Juvenile Osteology, London.

SCHOUR, I. y MASSLER, M. (1941): «The development of the human dentition». The Journal of the American dental Association 28, pp. 1153-1160

UBELAKER, D.H. (2007): «Enterramientos humanos: excavación, análisis, interpretación». Munibe suplemento, 24. Sociedad de Ciencias Aranzadi, Gehigarria. 